\title{
Preliminary Evaluation of QuantiFERON SARS-CoV-2 and QIAreach Anti-SARS-CoV-2 Total Test in Recently Vaccinated Individuals
}

Soumya Jaganathan

QIAGEN Sciences Inc

Francis Stieber

QIAGEN Sciences Inc

Sonia N. Rao ( $\nabla$ sonia.rao@qiagen.com )

QIAGEN Inc.

Vladyslav Nikolayevskyy

QIAGEN Manchester Ltd

Davide Manissero

QIAGEN Manchester Ltd

Nadia Allen

QIAGEN Sciences Inc

Jeff Boyle

QIAGEN Sciences Inc

Jenny Howard

QIAGEN Sciences Inc

\section{Research Article}

Keywords: Cell-mediated immunity, COVID-19, QuantiFERON, SARS-CoV-2, T-cell mediated response, Vaccination

Posted Date: June 21st, 2021

DOI: https://doi.org/10.21203/rs.3.rs-623620/v1

License: (c) (i) This work is licensed under a Creative Commons Attribution 4.0 International License.

Read Full License 


\section{Abstract}

Introduction: There is an increasing body of evidence surrounding the importance of a T-cell mediated response to SARS-CoV-2 infection and after COVID-19 vaccination. In this internal feasibility study, we evaluated both the total antibody (IgA, IgM, and IgG) and T-cell responses in a cohort of COVID-19 convalescents and vaccinated individuals.

Methods: Whole blood specimens were collected weekly from 12 subjects at different timepoints within/after the COVID-19 mRNA vaccination regimen and from 4 PCR-confirmed convalescent donors to measure durability of humoral and cell-mediated immune response. T-cell and Antibody responses were evaluated via the QuantiFERON SARS-CoV-2 Research Use Only (QFN SARS-CoV-2) assay which is an interferon gamma release assay (IGRA) and QIAreach Anti-SARS-CoV-2 Total (Anti-CoV-2) Test, respectively.

Results: In a cohort of recently vaccinated individuals, subjects demonstrated robust total antibody and CD4+/CD8+ T-cell response to SARS-CoV-2 mRNA vaccines when followed for 2 months post $2^{\text {nd }}$ dose. In most individuals, T-cell response declined between $1^{\text {st }}$ and $2^{\text {nd }}$ dose suggesting need for booster or completion of the 2-dose vaccine series. In a group of convalescent donors tested with QFN SARS-CoV-2 and Anti-CoV-2 tests, all patients had an antibody and T-cell response up to 1 year after natural infection.

Conclusion: This small feasibility study demonstrates that QFN-SARS-CoV-2 test is able to identify CD4+ and CD8+ T-cell mediated responses in SARS-CoV-2 vaccinated subjects and those recovered from COVID-19, alongside a qualitative antibody response detectable via the QIAreach Anti-CoV2 Test.

\section{Introduction}

The coronavirus disease 2019 (COVID-19) pandemic caused by the novel severe acute respiratory syndrome coronavirus-2 (SARS-CoV-2) has resulted in significant global morbidity and mortality with far reaching health and economic implications [1]. Vaccines that provide durable immunity to SARSCoV2 are a crucial component of the strategy to combat COVID-19 [2]. To achieve the desired durable immunity, a vaccine should not only evoke a robust production of neutralising antibodies ( $\mathrm{nAb}$ ), but also evoke a strong CD4 + and CD8 + T-cell response [3].

There is an increasing body of evidence surrounding the importance of a T-cell mediated response to SARS-CoV-2 infection. Peripheral T-cell lymphopenia appears to correlate with increased COVID-19 disease severity [4], whilst COVID-19 recovery is often associated with the presence of reactive CD4 + and CD8 + T-cells $[5,6]$. Robust evidence on long-term immunity is lacking, but it appears likely that a Tcell response is sustained for several months after infection and may last longer than a detectable antibody response [7, 8]. Past experience from the 2003 SARS-CoV-1 epidemic supports this, as initial specific immunoglobulin (Ig) G and $n A b$ response to SARS-CoV-1 diminished 2-3 years after infection [9], whilst Tcell memory was detected up to 11 years after infection [10]. Evidence also indicates that T-cell response is less likely to be affected by SARS-CoV-2 variants than antibody response $[11,12]$. In a recent study, 
Geers et al. demonstrated that the B.1.1.7 and B.1.351 variants of concern were able to partially evade humoral immunity, but that CD4 + T-cell activation was unaffected [12].

In addition to the importance of T-cell response to natural SARS-CoV-2 infection, there is a significant need to research T-cell responses to COVID-19 vaccination. In phase 1/2 trials, the mRNA1273, BNT162b2, ChAdOx1 and Ad26.COV2.S vaccines were all associated with a specific Tcell response to vaccination, at time points ranging from 7-43 days after first vaccination dose [13-16]. Kalimuddin recently reported a T-cell response measured by IFN- $y$ secretion in 19/20 subjects 10 days after a single dose of the BNT162b2 vaccine, before a nAb response was detected [17]. This led the authors to conclude that an early T-cell response in vaccinated individuals may play a greater role than nAb in generating early protection against COVID19 [17]. Interestingly, Parry recently reported a detectable T-cell response in $12.3 \%$ and $30.7 \%$ of older donors ( $\geq 80$ years), 5 weeks after a single dose of BNT162b2 or ChAdOx1 vaccine, respectively [18], suggesting T-cell responses may vary depending on vaccine and potentially may be reduced in older populations.

Therefore, in this small internal feasibility study, we evaluated both the total antibody (IgA, $\lg \mathrm{M}$, and $\lg G)$ response and T-cell response in a cohort of COVID-19 vaccinated individuals and convalescent donors. We hypothesized that SARS-CoV-2 mRNA vaccinated subjects will generate specific CD4 + and CD8 + Tcell mediated responses, detectable using the QuantiFERON® SARS-CoV-2 Research Use Only (QFN SARS-CoV-2) assay. Additional hypotheses for this study were that T-cell response will be detectable earlier than antibody response, as measured using the QIAreach Anti-SARS-CoV-2 Total Antibody (AntiCoV2) Test, and that higher responses may be seen after booster dose compared with initial vaccination dose.

\section{Methods}

\section{Samples}

Whole blood specimens were collected into lithium heparin tubes from 12 consenting subjects at different timepoints within/after the COVID-19 mRNA vaccination regimen and from 4 convalescent donors who recovered from PCR-confirmed COVID-19. The specimens collected from these subjects were shared between the QFN SARS-CoV-2 assay and the Anti-CoV2 Test. Once a subject was enrolled in the study, specimens were collected weekly immediately after the first dose and until at least six weeks following the second vaccination dose to measure durability of humoral and cell-mediated immune response.

Antibody response was measured using the Emergency Use Authorization (EUA) granted Anti-CoV2 Test, a digital lateral flow serological test using patented nanoparticle fluorescence technology that qualitatively detects total antibodies to SARS-CoV-2 in human serum and plasma. For this assay, the specimens in lithium heparin tubes were first centrifuged for 15 minutes at 2500 RCF to separate plasma from cells and facilitate sample harvesting. For each subject specimen, plasma was harvested from the 
lithium heparin tube and run with Anti-CoV2 according to the instructions for use. Qualitative test results and time-to-result for Anti-CoV2 positive subjects were recorded.

The T-cell response was evaluated via the QFN SARS-CoV-2 assay which is an interferon gamma release assay (IGRA). This assay consists of three Antigen tubes, SARS-CoV-2 Ag1, Ag2 and Ag3, that use a combination of proprietary antigen peptides specific to SARS-CoV-2 to stimulate lymphocytes involved in cell-mediated immunity in heparinized whole blood. The QFN SARS CoV-2 Ag1 tube contains CD $4+$ epitopes derived from the $\mathrm{S} 1$ subunit (Receptor Binging Domain) of the Spike protein, the Ag2 tube contains CD 4 + and CD8 + epitopes from the S1 and S2 subunits of the Spike protein and the Ag3 tube consists of CD4 + and CD8 + epitopes from S1 and S2, plus immunodominant CD 8 + epitopes derived from whole genome. Plasma from the stimulated samples can be used for detection of IFN- $\gamma$ using an enzyme-linked immunosorbent assay (ELISA)-based platform. Specimens were processed as per Manufacturer's guidelines [19-22]. Following ELISA, quantitative results (IFN- $\gamma$ concentration in IU/ml) were recorded and used for analysis. Elevated response was defined as a value at least $0.20 \mathrm{IU} / \mathrm{mL}$ greater than the background IU/mL value from the QFN-SARS-CoV-2 Nil tube.

All subjects included in the study provided informed consent, and all blood collections and testing were performed by trained professionals at QIAGEN Sciences, Inc.

\section{Results}

\section{Subjects}

In total, 16 subjects were included: 12 subjects who received two doses of mRNA-1273 SARS-CoV-2 vaccine and 4 subjects diagnosed with COVID-19. Among the 12 vaccinated subjects, 2 (16.7\%) were male and 10 (83.3\%) were female. The median age for vaccinated subjects was 44.5 years. Following vaccination, results were obtained at regular weekly intervals when possible, up to 70 days post first vaccination $(n=3), 74$ days post first vaccination $(n=8)$ or 99 days post first vaccination $(n=1)$.

Among the 4 convalescent subjects, 3 (75\%) were male and 1 (25\%) was female. The median age for convalescent subjects was 53 years. The time between PCR-confirmed COVID-19 diagnosis and first plasma samples ranged 1-11 months, and all subjects had confirmed resolution of COVID-19 symptoms prior to donating plasma; one subject reported no symptoms during the entirety of COVID-19 illness (Table 1). 
Table 1

COVID-19 disease information convalescent subjects providing samples for testing on QFN-SARS-CoV-2

\begin{tabular}{|llll|}
\hline $\begin{array}{l}\text { Subject } \\
\text { ID }\end{array}$ & $\begin{array}{l}\text { Symptom } \\
\text { onset }\end{array}$ & $\begin{array}{l}\text { COVID-19 } \\
\text { diagnosis date }\end{array}$ & $\begin{array}{l}\text { Symptom } \\
\text { end }\end{array}$ \\
\hline 1 & $1 / 10 / 2021$ & $1 / 5 / 2021$ & $1 / 12 / 2021$ \\
\hline 2 & $12 / 28 / 2020$ & $1 / 10 / 2021$ & $1 / 22 / 2021$ \\
\hline 3 & NA & $3 / 9 / 2020$ & $6 / 30 / 2020$ \\
\hline 4 & $9 / 25 / 2020$ & $9 / 26 / 2020$ & $10 / 20 / 2020$ \\
\hline $\begin{array}{l}\text { COVID-19 coronavirus disease 2019, QFN-SARS-COV-2 QuantiFERON } B \text { severe acute respiratory } \\
\text { syndrome coronavirus-2 }\end{array}$ \\
\hline
\end{tabular}

One negative control was included in the study, a non-vaccinated subject with non-reactive COVID-19 serology test results. Results were obtained for this subject at regular intervals between January 26 and March 2, 2021. The subject received Ad26.COV2.S COVID-19 vaccine (Johnson \& Johnson, NJ, USA) on March 6, 2021. Results post-vaccination for this subject were not analyzed in this study.

\section{QFN SARS-CoV-2 Assay in Vaccinated Subjects}

An elevated IFN-y response in at least one Ag tube was observed in the majority of vaccinated subjects (10/12) following first dose, in all subjects after the second dose, and in most subsequent time points (Fig. 1). Most subjects had elevated Ag responses sustained through the study, including one subject with a sample 70 days after booster dose.

The median (min-max) Nil subtracted IFN-y response for the 12 vaccinated subjects following initial vaccination dose and immediately prior to the booster dose (median 25 days, range 22-28 days) was 0.13 (-0.02-1.76), $0.20(0.02-1.88)$ and $0.25 \mathrm{IU} / \mathrm{mL}(0.01-2.43)$ for Ag1, Ag2 and Ag3 tubes, respectively. The median (min-max) Nil subtracted Ag1, Ag2 and Ag3 IFN-y responses at four weeks (median 32 days, range 28-32 days) post-second dose for 11 vaccinated subjects were $0.70(-0.27-$ $3.67), 0.90(0.07-5.17)$ and $1.15(0.11-5.75) \mathrm{IU} / \mathrm{mL}$, respectively, compared with $0.00,0.00$ and 0.00 $\mathrm{IU} / \mathrm{mL}$ in the nonreactive control subject. One of the vaccinated subjects was unavailable for testing at 4 weeks following the booster dose.

Following the initial mRNA-1273 vaccination dose, QFN SARS-CoV-2 responses peaked at 11-14 days, with median Nil subtracted responses of $1.29(0.04-4.76), 3.46(0.07-8.40)$ and $3.08(0.03-9.17) \mathrm{IU} / \mathrm{mL}$ for Ag1, Ag2 and Ag3, respectively, then steadily decreased until reaching $0.10(-0.02-1.76), 0.17(0.02-$ 1.88 ) and $0.26(0.01-2.43) \mathrm{IU} / \mathrm{mL}$ at $25-28$ days immediately prior to, or just after, receiving the booster dose (Fig. 1C). QFN SARS-CoV-2 responses spiked again 7-11 days after the vaccine booster dose, with median Nil subtracted responses of 2.34 (0.38-6.84), $2.96(0.46-6.22)$ and $4.42(0.33-9.93) \mathrm{IU} / \mathrm{mL}$ for $\mathrm{Ag} 1, \mathrm{Ag} 2$ and Ag3 tubes, respectively, then trended downwards to $0.70(-0.27-3.67), 0.90(0.07-5.17)$ and $1.15(0.11-5.75) \mathrm{IU} / \mathrm{mL}$ at $28-32$ days following the booster dose. 
Across all vaccinated subjects, higher responses were observed in Ag3 tubes than Ag2 tubes, and in Ag2 tubes than Ag1 tubes. In a non-parametric Wilcoxon signed rank test comparison between paired Ag1-Nil and Ag2-Nil responses performed separately for each of 4 weeks following the vaccine booster dose, Ag2Nil responses were consistently higher than Ag1-Nil responses (Fig. 1a). Likewise, for the same comparison between paired Ag3-Nil and Ag2-Nil responses, Ag3-Nil responses were higher for 3 of 4 weeks of testing post booster dose (Fig. 1b).

Longitudinally, no elevated responses were recorded on Day 4 after first dose, but all subjects had elevated responses by Day 11 (Fig. 1c). Among two subjects with samples at Day 7 post-first dose, both had elevated (Nil subtracted) responses: $0.27 / 0.61,0.39 / 0.33$ and $0.29 / 0.80 \mathrm{IU} / \mathrm{mL}$ for the Ag1, Ag2 and Ag3 tubes, respectively.

\section{QIAreach Anti-CoV2 Test in Vaccinated Subjects}

All subjects had detectable SARS-CoV-2 antibodies, as measured in their last sample in the study; average time-to-result (TTR) was slower in earlier samples post-first dose (Fig. 2). Among seven subjects with consecutive Day 4 and 11 samples post-first dose, there were no positive results at Day 4 , whereas all Day 11 samples were subsequently positive; median TTR was 3.4 minutes. No antibody response was detected in the two subjects tested at Day 7 post-first dose; both subjects had positive samples in their subsequent Day 14 samples.

\section{Convalescent Subjects}

All samples provided by convalescent subjects were serologically positive by the QIAreach AntiCoV2 Test at all test points, including initial baseline testing that occurred at least 1 month following COVID-19 diagnosis. Elevated QFN SARS-CoV-2 Ag1 and Ag2 IFN- $\gamma$ responses were detected throughout sampling in $3 / 4$ subjects, equating to approximately 1,5 , and 11 months post COVID-19 diagnosis (Table 1 ). IFN- $\gamma$ responses over time were broadly level (Fig. 3). In one subject who had no detectable responses at the start of sampling, responses were elevated in $\mathrm{Ag} 1$ and $\mathrm{Ag} 2$ in the last two samples, following vaccination. All of the subject samples had elevated IFN-y responses in Ag3.

\section{Discussion}

In this small internal feasibility study, QFN SARS-CoV-2 assay detected both CD $4+$ and CD8 + T-cellmediated responses in whole blood samples from subjects vaccinated with mRNA-1273 SARS-CoV-2 vaccine. Elevated IFN-y were observed in all three of the antigen tubes, suggesting all are able detect a Tcell mediated response. In a cohort of convalescent plasma subjects, $3 / 4$ subjects had elevated IFN-y responses.

In the majority of subjects, detectable antibody and elevated T-cell response to vaccination occurred simultaneously. As TTR is directly proportional to the amount of SARS-CoV-2 antibodies present (minimum TTR of 3 minutes for high antibody responses and 10 minutes for a low concentration 
antibody response), the median time to antibody results of 3.4 minutes at Day 11 implies an elevated humoral response that occurred alongside the elevated T-cell responses also detected on that day. However, antibody test results were negative for the two subjects tested at Day 7, whilst Ag1, Ag2 and Ag3 responses were detected in both. This suggests that a T-cell-mediated response to vaccination may occur prior to an antibody response, between Day 5 and Day 7. Phase 1 trials of the Moderna vaccine only assessed T-cell response at Days 1, 29, and 43 after initial vaccination [23], likely obtaining results several weeks after the T-cell response was initiated.

Longitudinally, the QFN SARS-CoV-2 assay detected a decline in T-cell-mediated response following the first vaccine dose, followed by a subsequent increase following second-dose vaccination. In multiple subjects, second-dose vaccination elicited an augmented Ag1, Ag2 and Ag3 response compared with the response to initial dose. Furthermore, an augmented response was seen in many of the Ag2 tubes compared with Ag1 tubes, indicating that both $\mathrm{CD} 4+$ and $\mathrm{CD} 8+\mathrm{T}$-cells contributed to the T-cell response detected. Similarly, the BNT162b2 vaccine has been shown to elicit strong CD $4+$ and CD $8+$ responses [14]. The Ag3 tube, which consisted of a selection of immunodominant peptides to the whole SARS-CoV-2 genome, demonstrated a slightly higher response compared to Ag1 or Ag2 in some individuals. However, this may be due to the selection of peptides included in the Ag3 tubes, as all subjects received spikebased vaccines. In terms of a long-term humoral response, the consistently low TTR implies a strong and sustained total antibody response occurred throughout the study.

The results of this study are consistent with other studies investigating the immune response to SARSCoV-2 infection. Several studies demonstrated a T-cell mediated response to natural SARS-CoV-2 infection 3-5 days after the onset of symptoms [24,25], which was estimated to be roughly seven days after infection [26]. It has also been observed that in some patients, particularly those with asymptomatic or mild SARS-CoV-2 infection, a T-cell specific response is detectable even in the absence of a detectable antibody response $[25,27,28]$. In addition, numerous vaccine studies have demonstrated a selective T-cell response in patients vaccinated against SARS-CoV-2 [14, 15, 23]; Ewer reported that a T-cell mediated response was also detected as early as Day 7 , earlier than a serological response, in patients receiving the ChAd0x1 nCov-19 vaccine [15]. In a real-world study of care home residents and staff, $87 \%$ of COVID-19 naïve staff $(n=15)$ demonstrated a Th1 IFN-y response four weeks after first vaccination with BNT162b2 [29]. These results were achieved using a cutoff point of $0.15 \mathrm{IU} / \mathrm{ml}$ to define a Th1 IFN-y response, slightly lower than the $0.20 \mathrm{IU} / \mathrm{ml}$ used in this study to define an elevated T-cell response. However, the Tcell response observed in vaccinated, COVID-19 naïve residents was significantly lower than that observed in the staff $(48 \%, P<0.001)$. This suggests older recipients of the BNT162b2 vaccine may generate a lower or delayed immunological response to initial vaccination, which warrants further investigation in future studies.

Other vaccine studies have also demonstrated that the T-cell response following a booster vaccine dose is consistently higher than the response observed after initial vaccination [23]. In a Phase 1 trial of mRNA1273 SARS-CoV-2 vaccine, 45 patients were vaccinated on Day 1 and received a booster dose 28 days 
later, on Day 29. The T-cell response detected on Day 43 was frequently higher than the response detected on Day 29 [23].

In our current study, T-cell response was observable for the duration of the study, including in one subject 70 days post second-dose of vaccine. Similarly, sustained T-cell responses were observed in convalescent plasma from subjects up to 11 months post infection. Long-term data on T-cell responses post vaccination is currently lacking. A recent study demonstrated that T-cell responses detected in previouslyinfected individuals after one vaccine dose was equivalent to naïve individuals receiving two vaccine doses [30], suggesting that a long-lasting T-cell response occurs from natural infection. This would appear to match with the results of our current study, where a prolonged T-cell response was observed following booster vaccination in vaccinated subjects.

Hypothetically, assessing the speed and strength of T-cell responses to a SARS-CoV-2 vaccine, using diagnostic tools such as the QFN SARS-CoV-2, could potentially provide more insight into vaccine efficacy. Identifying patient populations who demonstrate a reduced T-cell response to vaccination or infection may help to predict which patients are most at risk of severe disease. Conversely, detection of Tcell response could be particularly useful in selected populations of patients known to have impaired nAb/B-cell functionality. For example, Soresina et al. described two patients with X-linked agammaglobulinemia, who despite presenting with pneumonia after contracting COVID-19, did not require oxygen supplementation [31], indicating that cellular response may limit disease severity. Similarly, a study of patients with impaired humoral immunity due to hematologic cancer found CD $8+T-$ cell count was associated with increased survival and lower viral load [32]. In vulnerable patients such as these, identifying specific T-cell responses to vaccination could improve our understanding of vaccine efficacy and aid in stratifying patients by risk of severe disease.

Both antibody and T-cell responses decline after initial peaks following infection and vaccination [26], leaving uncertainty over the duration of immunity that vaccines provide. In the months following vaccination, QFN SARS-CoV-2 could be used to detect T-cell responses even when antibody levels are low or undetectable. As it appears likely that prior COVID-19 protects against reinfection, even in the absence of detectable antibodies [33], testing for T-cell response to vaccination could provide clearer information on vaccine-generated immunity, as opposed to testing antibody response alone. Furthermore, over a year into the COVID-19 pandemic, emerging vaccine-resistant variants are a concern and will likely require the adaptation of existing vaccines or development of new vaccines [34,35]. It is possible that T-cell response is less likely to be affected by virus variants than antibody response [11, 12], highlighting the potential utility of measuring T-cell responses in identifying promising vaccine candidates for the future.

This study is limited by its small testing cohort, frequency of sample collection and relatively short duration. Whilst the study does demonstrate the ability of QFN SARS-CoV-2 to generate an elevated T-cell response, the small number of subjects makes it difficult to draw strong conclusions based on these results. Additionally, one of the key questions surrounding SARS-CoV-2 vaccines is the duration of immunity generated. Whilst our results suggest QFN SARS CoV-2 is a viable method of quantifying T-cell 
response, even in those whose antibody response may have waned over time, further studies to evaluate T-cell responses several months after vaccination are warranted. Finally, this study only investigated T-cell responses to one mRNA SARS-CoV-2 vaccine (mRNA-1273), meaning conclusions cannot be drawn more widely for other vaccines.

\section{Conclusions}

This small feasibility study demonstrates that QFN SARS-CoV-2 test is able to identify CD4 + and CD8 + Tcell mediated responses in SARS-CoV-2 vaccinated subjects, alongside a qualitative antibody response detectable via the QIAreach Anti-CoV2 Test. While the T-cell and antibody response occurred simultaneously in most subjects, this study suggests T-cell responses may occur prior to an antibody response in some vaccinated subjects. The importance of a T-cell mediated response to vaccination has recently received more recognition and QFN SARS-CoV-2 offers an easy to use, viable method to assess this in vaccinated patients.

\section{Declarations}

This study protocol and documentation was approved by Advarra, an independent Institutional Review Board. The study authors declare no competing interests.

\section{Funding}

The study was funded by QIAGEN Manchester Ltd., Manchester, UK

\section{Authorship}

All named authors meet the International Committee of Medical Journal Editors (ICMJE) criteria for authorship for this article, take responsibility for the integrity of the work as a whole, and have given their approval for this version to be published.

\section{Medical Writing, Editorial, and Other Assistance}

Medical writing support for the development of this manuscript, under the direction of the authors and in accordance with Good Publications Practice (GPP3) guidelines (http://www.ismpp.org/gpp3) was provided by lan McAllister, BSc, of Ashfield MedComms, an Ashfield Health company, part of UDG Healthcare plc, for medical writing support that was funded by QIAGEN Manchester Ltd., Manchester, UK.

\section{Disclosures}

SJ is a former employee of QIAGEN Sciences Inc, NA is an employee of QIAGEN Sciences Inc, FS is an employee of QIAGEN Sciences Inc, SR is an employee of QIAGEN Inc, VN is an employee of QIAGEN Manchester Ltd, DM is an employee of QIAGEN Manchester Ltd, JB is a former employee of QIAGEN Sciences Inc and JH is an employee of QIAGEN Sciences Inc. 


\section{References}

1. World Health Organization (WHO). Coronavirus (COVID-19) Dashboard. 2021. https://covid19.who.int/. Accessed June 2021.

2. World Health Organization (WHO). COVID-19 vaccines. 2021. https://www.who.int/emergencies/diseases/novel-coronavirus-2019/covid-19-vaccines. Accessed June 2021.

3. Sauer K, Harris T. An Effective COVID-19 Vaccine Needs to Engage T Cells. Front Immunol. 2020;11:581807.

4. Shrotri M, van Schalkwyk MCl, Post N, et al. T cell response to SARS-CoV-2 infection in humans: A systematic review. PLoS One. 2021;16(1):e0245532.

5. Braun J, Loyal L, Frentsch M, et al. SARS-CoV-2-reactive T cells in healthy donors and patients with COVID-19. Nature. 2020;587(7833):270-4.

6. Peng Y, Mentzer AJ, Liu G, et al. Broad and strong memory CD4 (+) and CD8 (+) T cells induced by SARS-CoV-2 in UK convalescent COVID-19 patients. bioRxiv. 2020:Preprint.

7. Schwarzkopf S, Krawczyk A, Knop D, et al. Cellular Immunity in COVID-19 Convalescents with PCRConfirmed Infection but with Undetectable SARS-CoV-2-Specific IgG. Emerging infectious diseases. 2021;27(1).

8. Zuo J, Dowell AC, Pearce $\mathrm{H}$, et al. Robust SARS-CoV-2-specific T cell immunity is maintained at 6 months following primary infection. Nat Immunol. 2021;22(5):620-6.

9. Cao WC, Liu W, Zhang PH, Zhang F, Richardus JH. Disappearance of antibodies to SARS-associated coronavirus after recovery. N Engl J Med. 2007;357(11):1162-3.

10. Ng OW, Chia A, Tan AT, et al. Memory T cell responses targeting the SARS coronavirus persist up to 11 years post-infection. Vaccine. 2016;34(17):2008-14.

11. Tarke A, Sidney J, Methot N, et al. Negligible impact of SARS-CoV-2 variants on CD4 (+) and CD8 (+) T cell reactivity in COVID-19 exposed donors and vaccinees. bioRxiv. 2021:Preprint.

12. Geers D, Shamier MC, Bogers S, et al. SARS-CoV-2 variants of concern partially escape humoral but not T-cell responses in COVID-19 convalescent donors and vaccinees. Sci Immunol. 2021;6(59):eabj1750.

13. Anderson EJ, Rouphael NG, Widge AT, et al. Safety and Immunogenicity of SARS-CoV-2 mRNA-1273 Vaccine in Older Adults. N Engl J Med. 2020;383(25):2427-38.

14. Sahin U, Muik A, Vogler I, et al. BNT162b2 induces SARS-CoV-2-neutralising antibodies and T cells in humans. medRxiv. 2020:Preprint.

15. Ewer KJ, Barrett JR, Belij-Rammerstorfer S, et al. T cell and antibody responses induced by a single dose of ChAdOx1 nCoV-19 (AZD1222) vaccine in a phase 1/2 clinical trial. Nat Med. 2021;27(2):270-8.

16. Sadoff J, Le Gars M, Shukarev G, et al. Interim Results of a Phase 1-2a Trial of Ad26.COV2.S Covid19 Vaccine. N Engl J Med. 2021;384(19):1824-35. 
17. Kalimuddin S, Tham CY, Qui M, et al. Early T cell and binding antibody responses are associated with Covid-19 RNA vaccine efficacy onset. Med (N Y). 2021:doi: 10.1016/j.medj.2021.04.003 [Epub ahead of print].

18. Parry HM, Bruton R, Tut G, et al. Single Vaccination with BNT162b2 or ChAdOx1 in Older People Induces Equivalent Antibody Generation but Enhanced Cellular Responses after ChAdOx1. Lancet. 2021:Preprint at SSRN: https://ssrn.com/abstract=3825573.

19. Qiagen. QuantiFERON® SARS-CoV-2 Extended Set Blood Collection Tubes Instructions for Use (Handbook). April 2021. https://www.qiagen.com/us/. Accessed June 2021.

20. Qiagen. QuantiFERON® Control Set Blood Collection Tubes Instructions for Use (Handbook). March 2021. https://www.qiagen.com/us/. Accessed June 2021.

21. Qiagen. QuantiFERON® SARSCoV-2 Starter Set Blood Collection Tubes Instructions for Use (Handbook). March 2021. https://www.qiagen.com/us/. Accessed June 2021.

22. Qiagen. QuantiFERON® ELISA Instructions for Use (Handbook). March 2021. https://www.qiagen.com/us/. Accessed June 2021.

23. Jackson LA, Anderson EJ, Rouphael NG, et al. An mRNA Vaccine against SARS-CoV-2 - Preliminary Report. N Engl J Med. 2020;383(20):1920-31.

24. Rydyznski Moderbacher C, Ramirez SI, Dan JM, et al. Antigen-Specific Adaptive Immunity to SARSCoV-2 in Acute COVID-19 and Associations with Age and Disease Severity. Cell. 2020;183(4):9961012.e19.

25. Tan AT, Linster M, Tan CW, et al. Early induction of functional SARS-CoV-2-specific $T$ cells associates with rapid viral clearance and mild disease in COVID-19 patients. Cell Rep. 2021;34(6):108728.

26. Bertoletti A, Tan AT, Le Bert N. The T-cell response to SARS-CoV-2: kinetic and quantitative aspects and the case for their protective role. Oxford Open Immunology. 2021;2(1):iqab006.

27. Sekine T, Perez-Potti A, Rivera-Ballesteros 0 , et al. Robust T Cell Immunity in Convalescent Individuals with Asymptomatic or Mild COVID-19. Cell. 2020;183(1):158 - 68.e14.

28. Reynolds CJ, Swadling L, Gibbons JM, et al. Discordant neutralizing antibody and T cell responses in asymptomatic and mild SARS-CoV-2 infection. Sci Immunol. 2020;5(54):eabf3698.

29. Van Praet JT, Vandecasteele S, De Roo A, De Vriese AS, Reynders M. Humoral and cellular immunogenicity of the BNT162b2 mRNA Covid-19 Vaccine in nursing home residents. Clin Infect Dis. 2021:ciab300.

30. Angyal A, Longet S, Moore S, et al. T-Cell and Antibody Responses to First BNT162b2 Vaccine Dose in Previously SARS-CoV-2-Infected and Infection-Naive UK Healthcare Workers: A Multicentre, Prospective, Observational Cohort Study. Lancet. 2021:Preprint at SSRN: https://ssrn.com/abstract=3812375.

31. Soresina A, Moratto D, Chiarini $M$, et al. Two X-linked agammaglobulinemia patients develop pneumonia as COVID-19 manifestation but recover. Pediatr Allergy Immunol. 2020;31(5):565-9. 
32. Huang A, Bange $E$, Han N, et al. CD8 T cells compensate for impaired humoral immunity in COVID-19 patients with hematologic cancer. Res Sq. 2021:Preprint.

33. Breathnach AS, Duncan CJA, Bouzidi KE, et al. Prior COVID-19 protects against reinfection, even in the absence of detectable antibodies. J Infect. 2021;S0163-4453(21).

34. Madhi SA, Baillie V, Cutland CL, et al. Efficacy of the ChAdOx1 nCoV-19 Covid-19 Vaccine against the B.1.351 Variant. N Engl J Med. 2021;384(20):1885-98.

35. Walensky RP, Walke HT, Fauci AS. SARS-CoV-2 Variants of Concern in the United States-Challenges and Opportunities. JAMA. 2021;325(11):1037-8.

\section{Figures}

a

Ag1 vs Ag2 Responses Post Booster-Dose

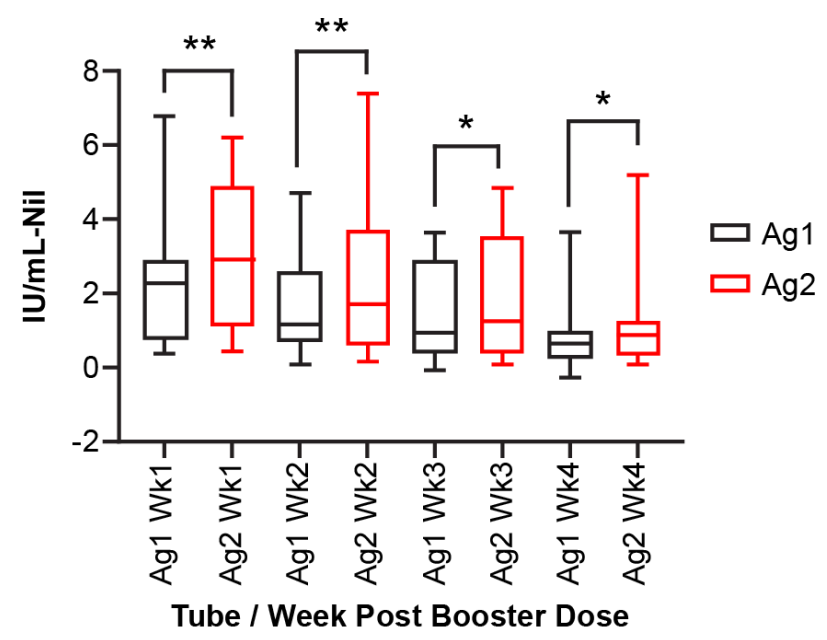

b

Ag2 vs Ag3 Responses Post Booster-Dose

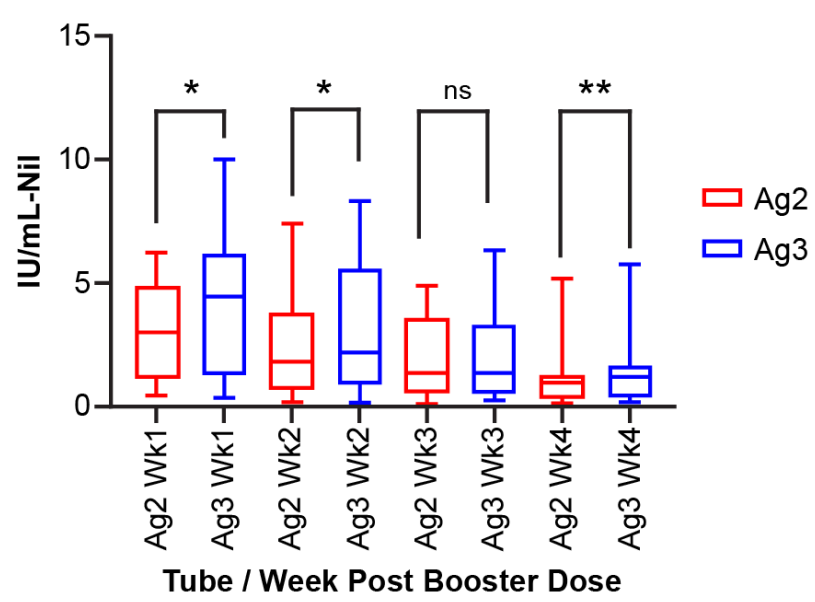

C
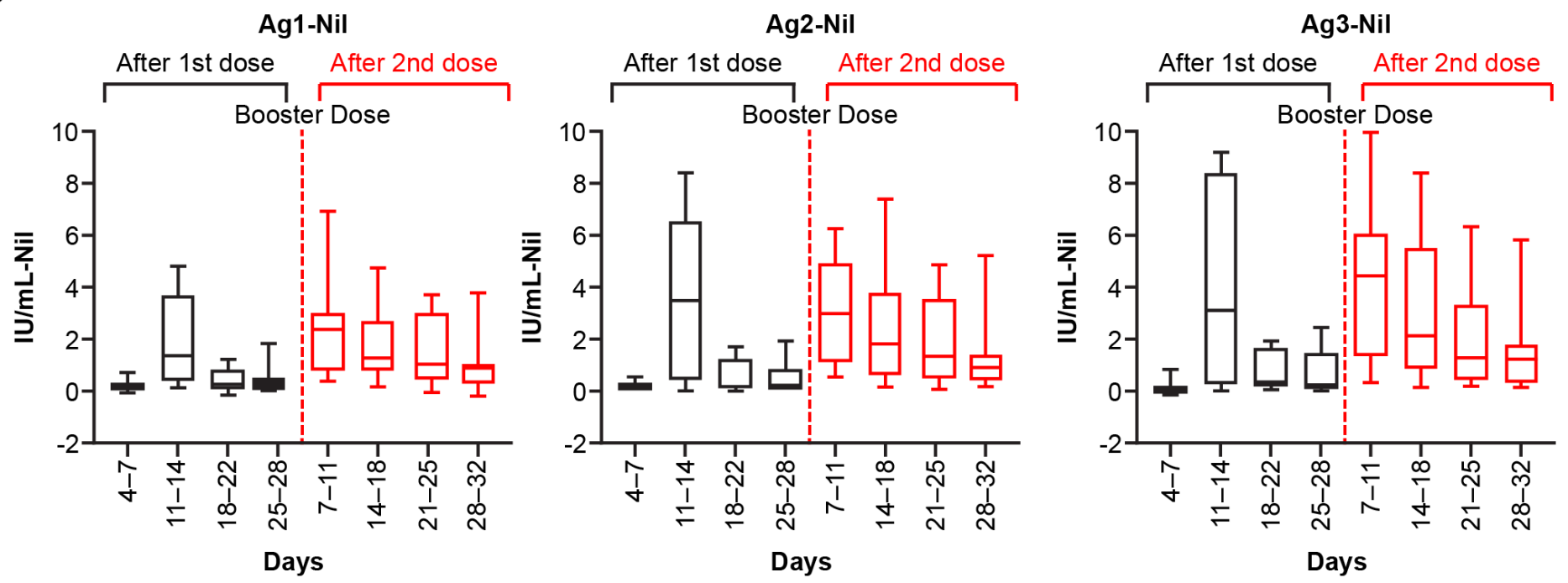

Figure 1 
Comparison of a QFN-SARS-CoV-2 Ag1-Nil and Ag2-Nil responses and b QFN-SARS-CoV-2 Ag2-Nil and Ag3-Nil responses in subjects vaccinated with the mRNA-1273 SARS-CoV-2 vaccine for four consecutive weeks post booster dose. Week 1: 12 subjects, median 11 days (7-11), Week 2: 12 subjects, median 18 days (14-18), Week 3: 11 subjects, median 25 days (21-25), Week 4, 11 subjects, median 32 days (2832). Responses are shown in a box plot with min-max range bars. Results from a Wilcoxon matched-pairs

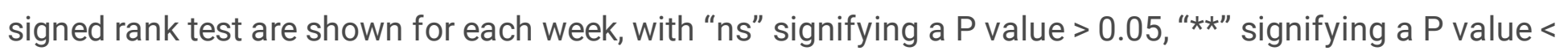
0.01 and " $*$ " signifying a $P$ value between 0.01 and 0.05 . c Longitudinal QFN-SARS-CoV-2 antigen tube (Ag minus Nil) response in subjects receiving both doses of the mRNA-1273 SARS-CoV-2 vaccine. Each date range contains a box plot with min-max range bars. Number of subjects: $4-7$ days (8), 11-14 days (10), 18-22 days (11), 25-28 days (11), 7-11 days (12), 14-18 days (12), 21-25 days (11), 28-32 days (11). 


\section{TTR Vaccinated Donors}

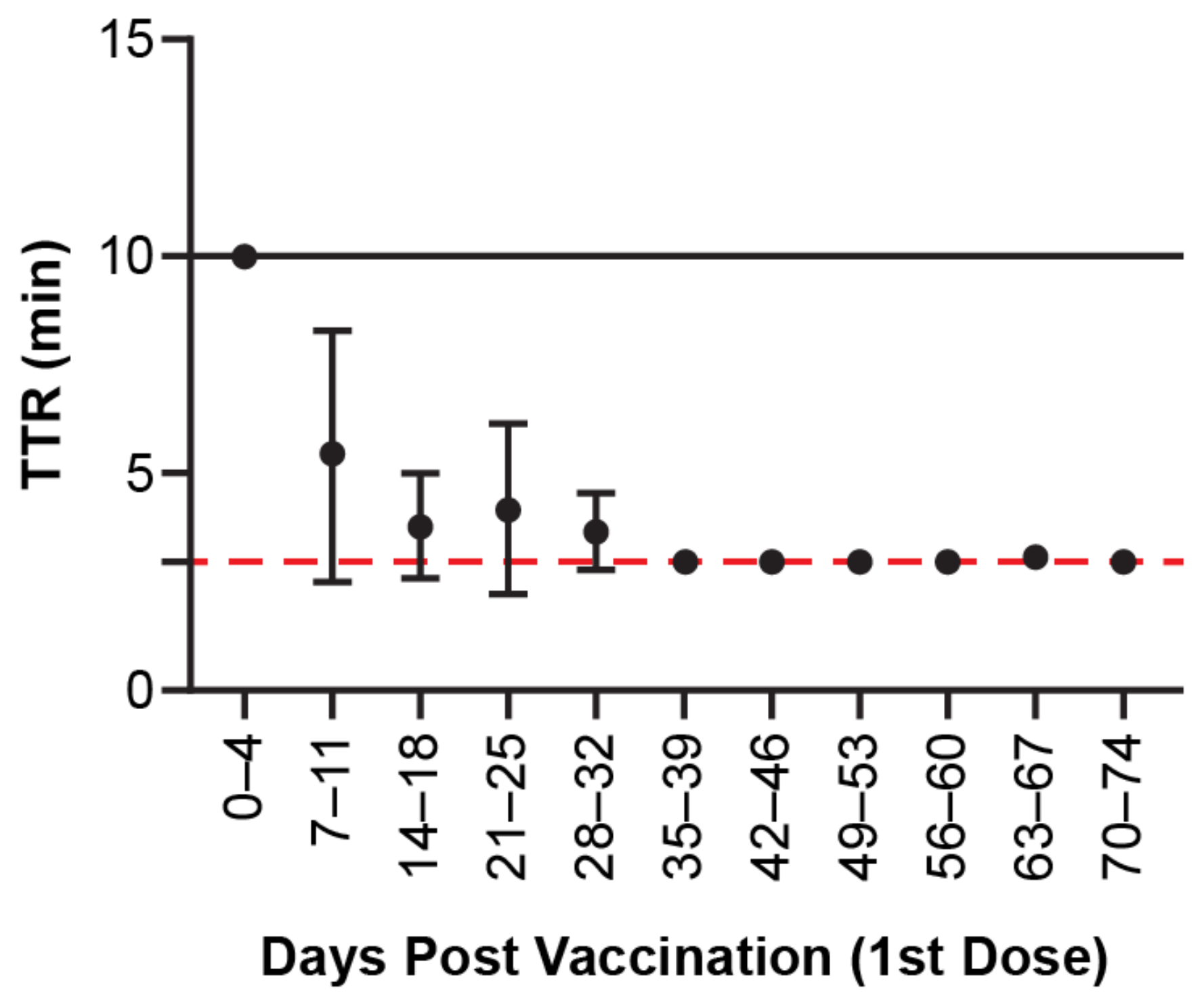

Figure 2

QIAreach Anti-SARS-CoV-2 Total Test (Anti-CoV2) time-to-result (TTR) in subjects vaccinated with mRNA1273 SARS-CoV-2 vaccine $(\mathrm{N}=12)$. For Anti-CoV2 testing, a result will be determined between 3-10 minutes. All negative tests will have a 10-min TTR. Positive antibody test results (detecting a total antibody response) will have a TTR between 3-10 minutes, with a lower TTR for higher levels of antibody detected by the device. All results following the 28-32 days post vaccination are from serology samples collected following the vaccine booster dose. 
a

Convalescent Ag1-Nil

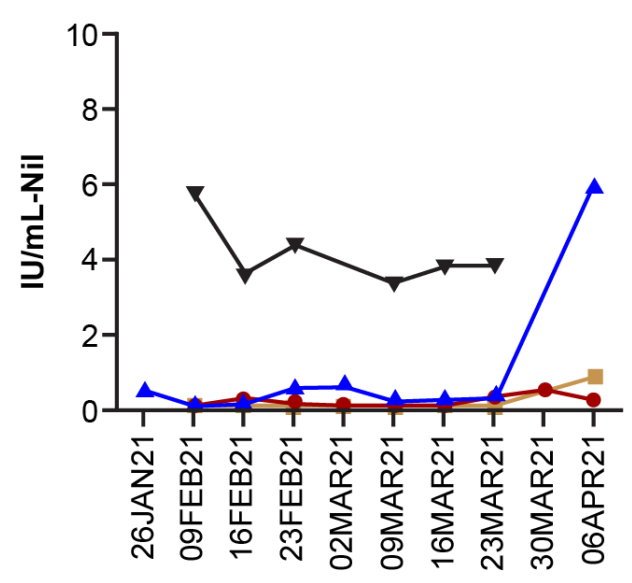

Date b

Convalescent Ag2-Nil

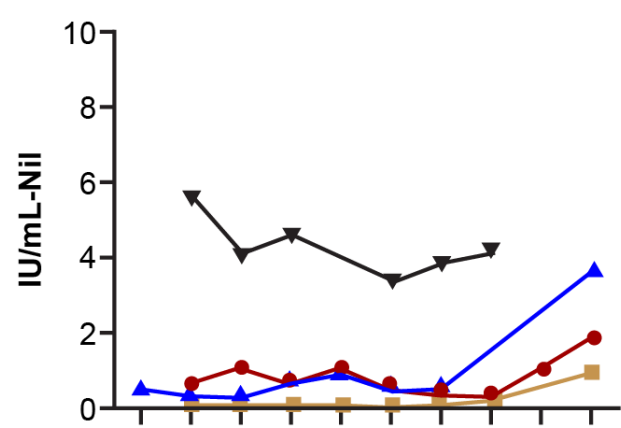

$\bar{\sim} \bar{\sim} \bar{\sim} \bar{\sim} \bar{N} \bar{N} \bar{N}$

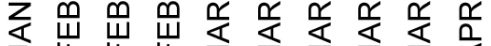

ঢ্থ

Date
C

Convalescent Ag3-Nil

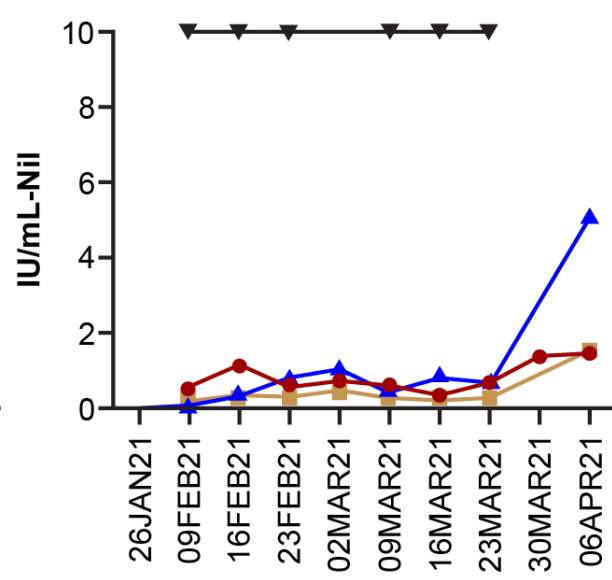

Date

Donor 1 -- Donor $2 \_$Donor $3-$ Donor 4

\section{Figure 3}

QFN-SARS-CoV-2 responses (Ag minus Nil) in Ag1 (a), Ag2 (b) and Ag3 (c) antigen tubes from convalescent subjects with prior COVID-19 diagnosis $(\mathrm{N}=4)$. 